\title{
Descripción ultraestructural de los diferentes estadios del ciclo de vida de Angiostrongylus costarricenses
}

\author{
Hernando Vargas Vargas*, María Victoria Torres*, Kevin González \\ Suárez** \\ 1. * Laboratorio Clínico del Hospital de Ciudad Neily, CCSS, Ciudad Neily, Costa Rica, \\ hernando2040@hotmail.com, marisa.victoria.torres@gmail.com \\ 2. **Laboratorio Clínico del Hospital de San Carlos, CCSS, Ciudad Quesada, Costa Rica, \\ mqc1665@gmail.com
}

Recibido: 16 de junio de 2020

Aceptado: 22 de octubre de 2020

\section{RESUMEN}

Angiostrongylus costaricensis es un nemátodo de importancia médica en América. En el presente estudio se realizó una descripción ultraestructural completa y precisa de A. costaricensis en cada uno de sus estadios de desarrollo. Se estudió un mínimo de 20 especímenes por estadio evolutivo los cuales se procesaron para microscopía de barrido y fueron observados y fotografiados a magnificaciones desde 25 a $3000 \mathrm{X}$.

Palabras claves: Angiostrongylus costaricensis, nematoda, ultraestructura, larvas, huevecillos

\section{ABSTRACT}

Angiostrongylus costaricensis is a nematode of medical importance in America. In the present study, a complete and precise ultrastructural description of A. costaricensis was made at each of its stages of development. A minimum of 20 specimens per evolutionary stage were studied which were processed for scanning microscopy and were observed and photographed at magnifications from 25 to $3000 \mathrm{X}$.

Key words: Angiostrongylus costaricensis, nematode, ultrastructure, larves, eggs,

\section{Introducción}

La familia Metastrongylidae comprende dos importantes géneros que afectan la salud humana, Metastrogylus y Angiostrongylus. Dentro del género Angiostrongylus se encuentran varias especies de importancia médica, entre las cuales encontramos a A. costaricensis (Stokes et al. 2007), el cual es objeto de este estudio ultraestructural.

Angiostrongylus costaricensis es una especie descrita por Morera y Céspedes en 1971, es capaz de producir en el ser humano un cuadro clínico patológico llamado angiostrongilosis abdominal (también angiostrongiliasis), que puede ser causante de formación de granulomas con intensa infiltración eosinofílica en la cavidad abdominal del paciente (Morera and Céspedesu 2002).

Este parásito se ha encontrado a lo largo del continente americano, con reportes desde el sur de los Estados Unidos de Norte América, hasta Argentina, con especial distribución en la zona del Caribe. (Valente et al. 2018). Su ciclo de vida es directo y heteroxeno obligado. El ciclo de vida natural involucra como hospedero intermediario a moluscos pulmonados terrestres de la familia Veronicellidae (Babosas), que ingieren las heces de los hospedadores definitivos conteniendo larvas L1, dentro de los cuales se desarrollan los estadios larvales de L1 a L3 (Banevicius et al. 2006). Los estadios adultos se desarrollan en arterias mesentéricas de la región ileocecal en roedores como Sigmodon hispidus, Ratus ratus y Oryzomys sp. que actúan como hospederos 
El ser humano contrae la infección al ingerir alimentos vegetales contaminados con secreciones de babosa en las que se encuentra la larva. Una vez ingerida, la larva infectante L3 madura durante su complejo viaje a través de diferentes tejidos hasta que coloniza el lumen de las arterias mesentéricas de la región ileocecal en donde se desarrolla el adulto y ocurre la oviposición. Es a este nivel que se produce la reacción inflamatoria que caracteriza a este nemátodo (Geiger et al. 2003).

La descripción morfológica de A. costaricensis fue realizada por Morera y Céspedes en 1971 utilizando microscopía de luz. Desde entonces se han realizado muy pocos estudios de este tipo que aporten o mejoren la información existente. Del mismo modo, existen escasos estudios ultraestructurales completos sobre el parásito en sus diferentes estadios, con excepción de las observaciones por (Rivera, Anrabel Araya; Lobo, Lucia Quesada; Vargas 2015). Este trabajo se desarrolló porque se considera que el conocimiento detallado de la morfología de este parásito es de utilidad para el diagnóstico y la taxonomía del mismo y para ayudar a solventar la escasez de información morfológica minuciosa referente a este helminto. Se tiene como objetivo describir con el mayor detalle posible, la ultraestructura de A. costaricensis en los diferentes estadios de su ciclo de vida y enriquecer la biblioteca de fotografías por microscopia electrónica existentes

\section{Metodología}

Obtención, almacenamiento y número de muestras de $A$. costaricensis.

Las cepas de Angiostrongylus costaricensis en sus diferentes estadios evolutivos utilizados en esta descripción fueron facilitados por los laboratorios de parasitología de la universidad de Costa Rica y del hospital San Juan de Dios a partir de ratas Sigmodon hispidus y de "babosas" Vaginulus plebeius, infectadas. Los especímenes fueron almacenados, en diferentes recipientes con alcohol de 70, según su estadio evolutivo. Para estudiar la ultraestructura de A. costaricensis se utilizaron cinco especímenes de cada uno de los estadios de desarrollo cultivables en laboratorio (huevecillos, estadios larvales L1 y L3 y formas adultas).

Preparación de muestras para microscopía electrónica de barrido.

El método de preparación de los diferentes estadios de desarrollo del parásito A. costaricensis para microscopía electrónica de barrido se efectuó según el procedimiento descrito por Sánchez y Espinoza (Sanchez and Espinoza 2005)(2005). En resumen, las muestras se fijan con glutaraldehído, se realiza una post- fijación con tetra óxido de osmio al 1\%, seguida de una deshidratación con incremento de gradiente de alcohol, se lleva a cabo un último pasaje a terbutanol. Seguidamente las muestras son secadas por sublimación (Kothari and Sagar 2008) son colocadas sobre discos de aluminio donde se recubren con un baño de platino. Después las muestras se secan en una incubadora a $37^{\circ} \mathrm{C}$ por 48 horas.

\section{Toma de microfotografías}

Las muestras fueron observadas mediante microscopía electrónica de barrido (modelo Hitachi S- 570). Las microfotografías se tomaron con una magnificación desde 25-3000X y se fotografiaron las estructuras de interés utilizando cámara digital.

\section{Resultados}

\section{Morfología general del adulto}

El cuerpo de ambos sexos se observó filiforme y adelgazado hacia el extremo anterior. La cabeza se presentó forma lisa y redondeada. En la boca no se observaron labios; pero sí se notó que estaba rodeada por anillos simétricos de 6 papilas y 2 grandes ánfides laterales (Fig. 1). El poro excretor se localizó anatómicamente entre el final del esófago y los intestinos. 


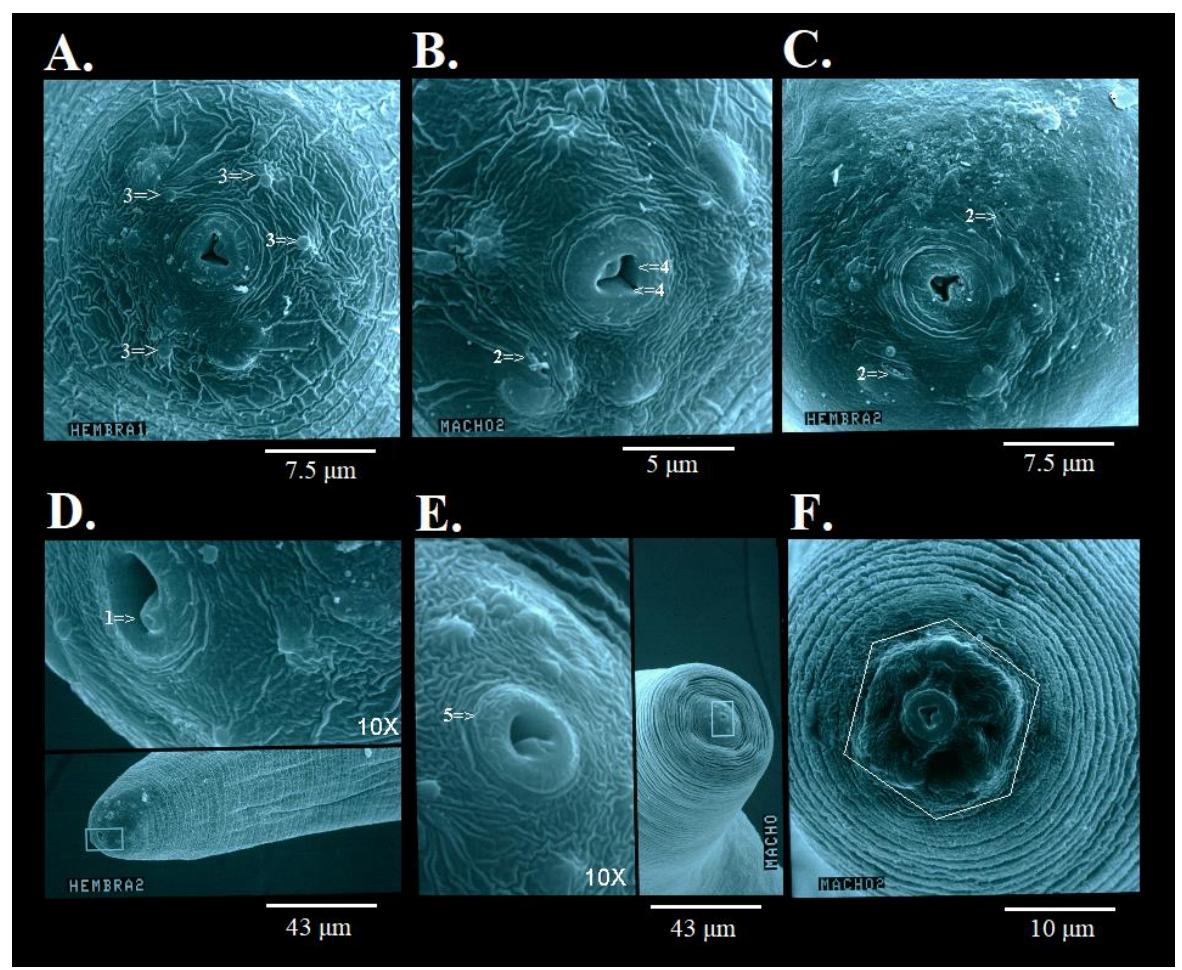

Figura 1. Cápsula bucal de adultos de Angiostrongylus costaricensis. A. Se señalan las papilas sensoriales, llamadas fásmides (3). B. Labios dentro de anillo oral (4). C. Ánfides (2) laterales opuestas mutuamente. D. Labio modificado en funda o cubierta. E. Anillo oral. F. Estructura hexaédrica de la cápsula bucal.

\section{Morfología del macho}

Se observó que la longitud de los machos fue de 17,3-19,4 mm, con una anchura en la base del esófago de 121-139 $\mu \mathrm{m}$, y una anchura máxima de 267-285 $\mu \mathrm{m}$. Las espículas se observaron similares, delgadas, y presentaron además un llamativo aspecto estriado y midieron 392-420 $\mu \mathrm{m}$ de longitud. Los extremos proximales de las espículas se mostraron con puntas irregulares, los extremos distales también se observaron punteados. El gubernáculo presentó una forma curvada, con una longitud de $42-54 \mu \mathrm{m}$.

La bolsa copulatriz presentó forma simétrica y bien desarrollada, con una pequeña incisión dorsal (Fig. 2). El rayo dorsal se observó corto, con dos largas ramas.

Las ramas externodorsales se observaron igual o aún más largas que las ramas dorsales, digitiformes y separadas en la base. Se observaron también los rayos ventrales y laterales, tal y como se muestra en Fig. 3.

Tabla 1. Medición de algunos parámetros en el adulto macho

\begin{tabular}{llllll}
\hline Muestra & Longitud de cuerpo $(\mathbf{m m})$ & $\begin{array}{l}\text { Anchura de base de esófago } \\
(\boldsymbol{\mu m})\end{array}$ & $\begin{array}{l}\text { Longitud de } \\
(\boldsymbol{\mu m})\end{array}$ & espículas \\
\hline 1 & 19,4 & 123 & & 411 & \\
2 & 19,1 & 121 & 417 & \\
3 & 17,5 & 132 & & 420 & \\
4 & 17,3 & 135 & 392 & \\
5 & 18,5 & 139 & 391 & \\
Promedio & 18,4 & 130 & & 406 & \\
\hline
\end{tabular}




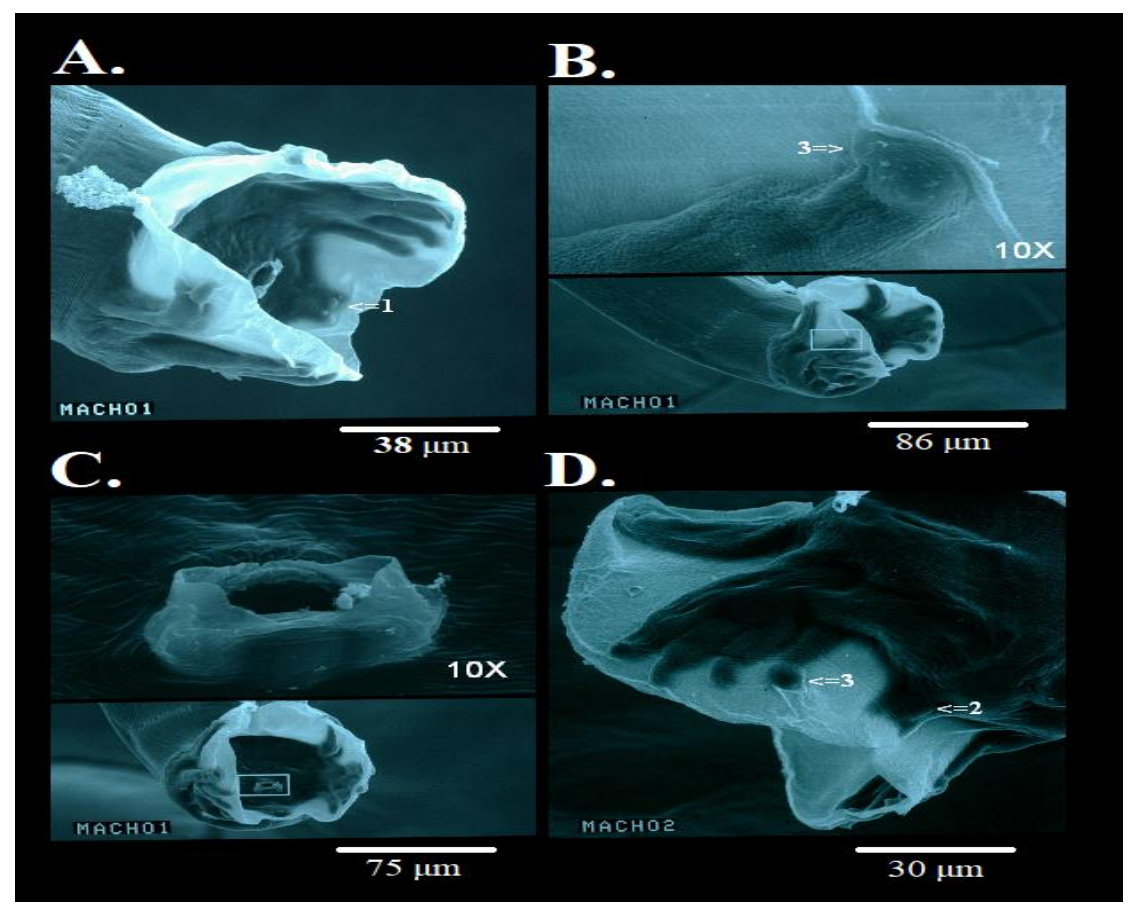

Figura 2. Genitalia de machos de Angiostrongylus costaricensis, Bursa copulatrix.

A. Se señalan los sacos sensoriales (1) sobre rayos caudales. B. Disposición característica de los rayos dorsales y acercamiento a rayo (3) evidenciando la globosidad terminal. C. Acercamiento cono genital. D. Vista dorsal señalándose globosidad terminal rayo (2) y abultamiento sobre rayos dorsales.

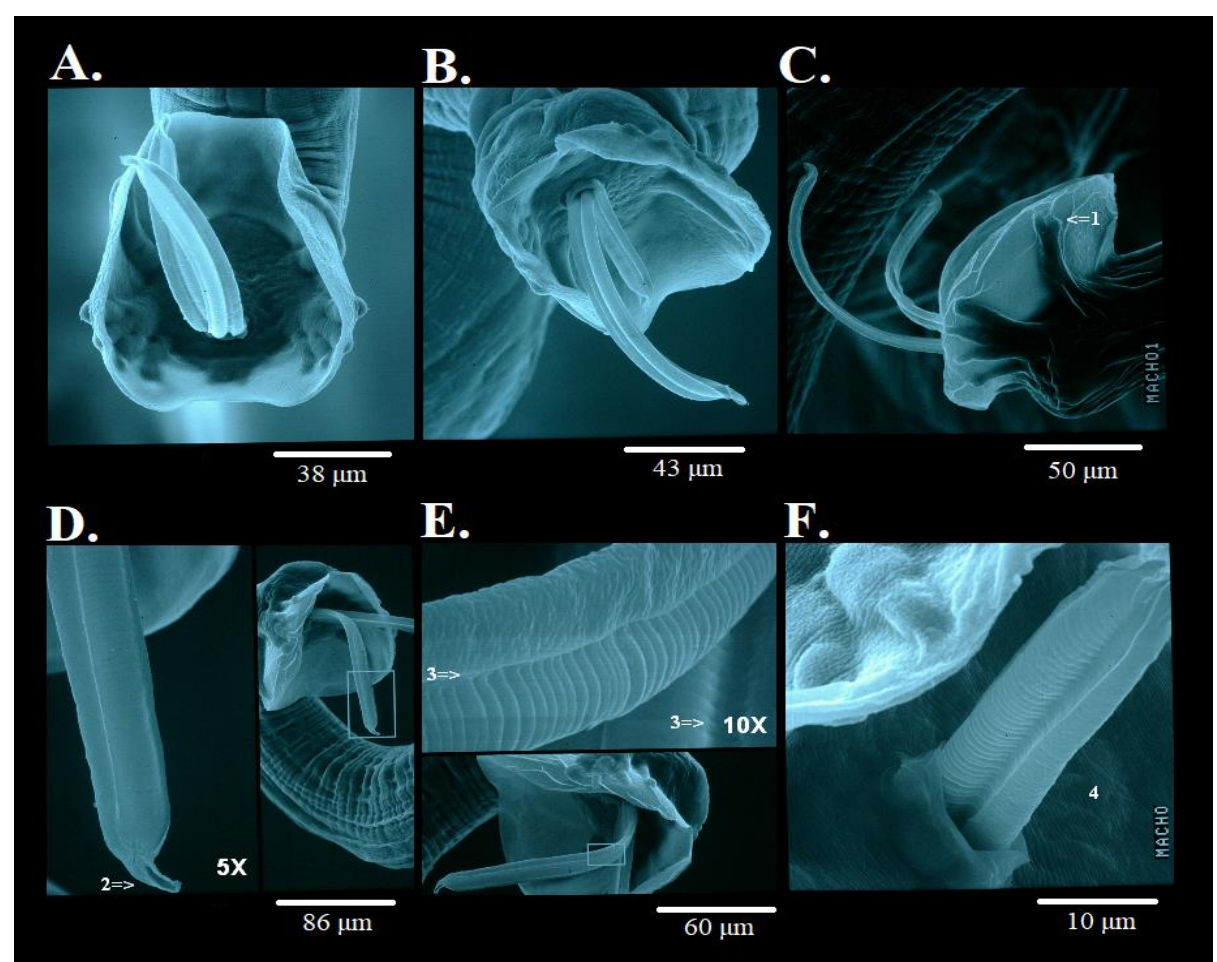

Figura 3. Genitalia de machos de Angiostrongylus costaricensis, Bursas copulatrices y espículas. A. Vista frontal. B. Evidencia la individualidad de movimiento de las espículas, de las cuales se observa una más salida que la otra. C. Vista lateral, espículas curveadas prominentes y rayo (1) dividido terminalmente (1). D. Acercamiento 5X especula en garfio acanalada (2). E. Acercamiento 10X cuerpo de la espícula canal (3) o depresión medial. F. Valle medial, bursa texturizada estrías circulares concéntricas (4) alrededor del cono genital. 


\section{Morfología de la hembra}

Se observó que la longitud de las hembras fue de 30,0-31,5 mm, con una anchura en la base del esófago de 170-175 $\mu \mathrm{m}$, y una anchura máxima de 288-335 $\mu \mathrm{m}$. En la vulva se observaron los labios a una distancia de 252-236 $\mu \mathrm{m}$ desde la punta de la cola. El ano, por su parte se localizó a 77-108 $\mu \mathrm{m}$ del extremo apical. La cola se mostró redondeada con una pequeña espiga en la punta.

Tabla 2. Medición de algunos parámetros en el adulto hembra.

\begin{tabular}{|l|l|l|l|}
\hline Muestra & $\begin{array}{l}\text { Longitud cuerpo } \\
(\mathrm{mm})\end{array}$ & $\begin{array}{l}\text { Anchura de base de esófago } \\
(\mu \mathrm{m})\end{array}$ & $\begin{array}{l}\text { Distancia de vulva con } \\
\text { respecto a la cola }(\mu \mathrm{m})\end{array}$ \\
\hline 1 & 30,0 & 172 & 250 \\
\hline 2 & 30,9 & 171 & 252 \\
\hline 3 & 30,8 & 175 & 239 \\
\hline 4 & 30,0 & 173 & 238 \\
\hline 5 & 31,5 & 170 & 243 \\
\hline Promedio & 30,6 & 172 & 236 \\
\hline
\end{tabular}

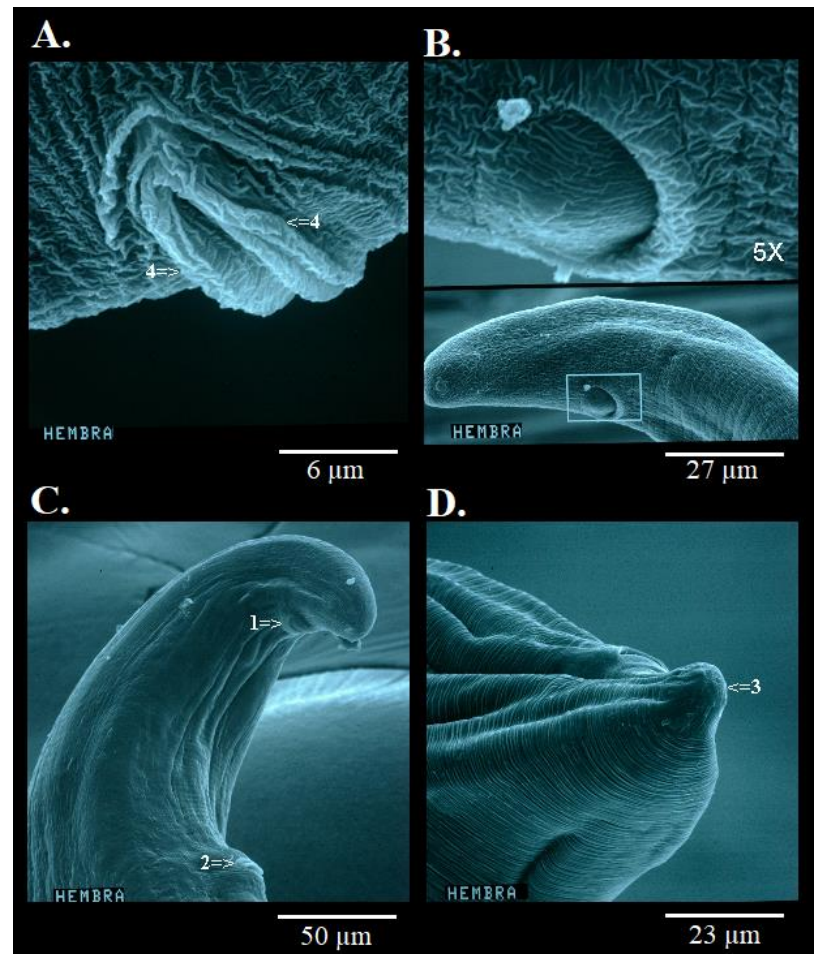

Figura 4. Genitalia de hembras de Angiostrongylus costaricensis. A. Vulva mostrando labios genitales(4). B. Hendidura anal, se muestra acercamiento a cinco aumentos. C. Vulva (2) y hendidura anal (1) a baja magnificación, observar la relación de tamaño y posición en la región posterior del nemátodo. D. Cauda mostrando espina (3) descrita por Morera; no representa el promedio de especímenes analizados.

\section{Morfología de la larva L3}

Se observó una larva filiforme que medía entre 0.46 a $0.482 \mathrm{~mm}$ de longitud y 28 a $30 \mu \mathrm{m}$ de ancho. La parte anterior del cuerpo es redonda y muestre unos abultamientos prominentes. Presentan cutícula bien definida, la cual tiene estriaciones transversales, expansiones cuticulares longitudinales y apertura oral mucho mejor definidas que las larvas L1. Se observó el desarrollo de una cápsula bucal bien definida, que recuerda a los adultos en cuanto a la presencia de labios y anillo oral, aunque no presentan el mismo grado de diferenciación que éstos (Figura 4).

En la cola se presenta un alargamiento dorsolateral a manera de alas o extensiones cuticulares (Figura $5)$. 
En la región caudal no se observó desarrollo de estructuras sexuales bien definidas y la muesca característica persiste en menor grado.

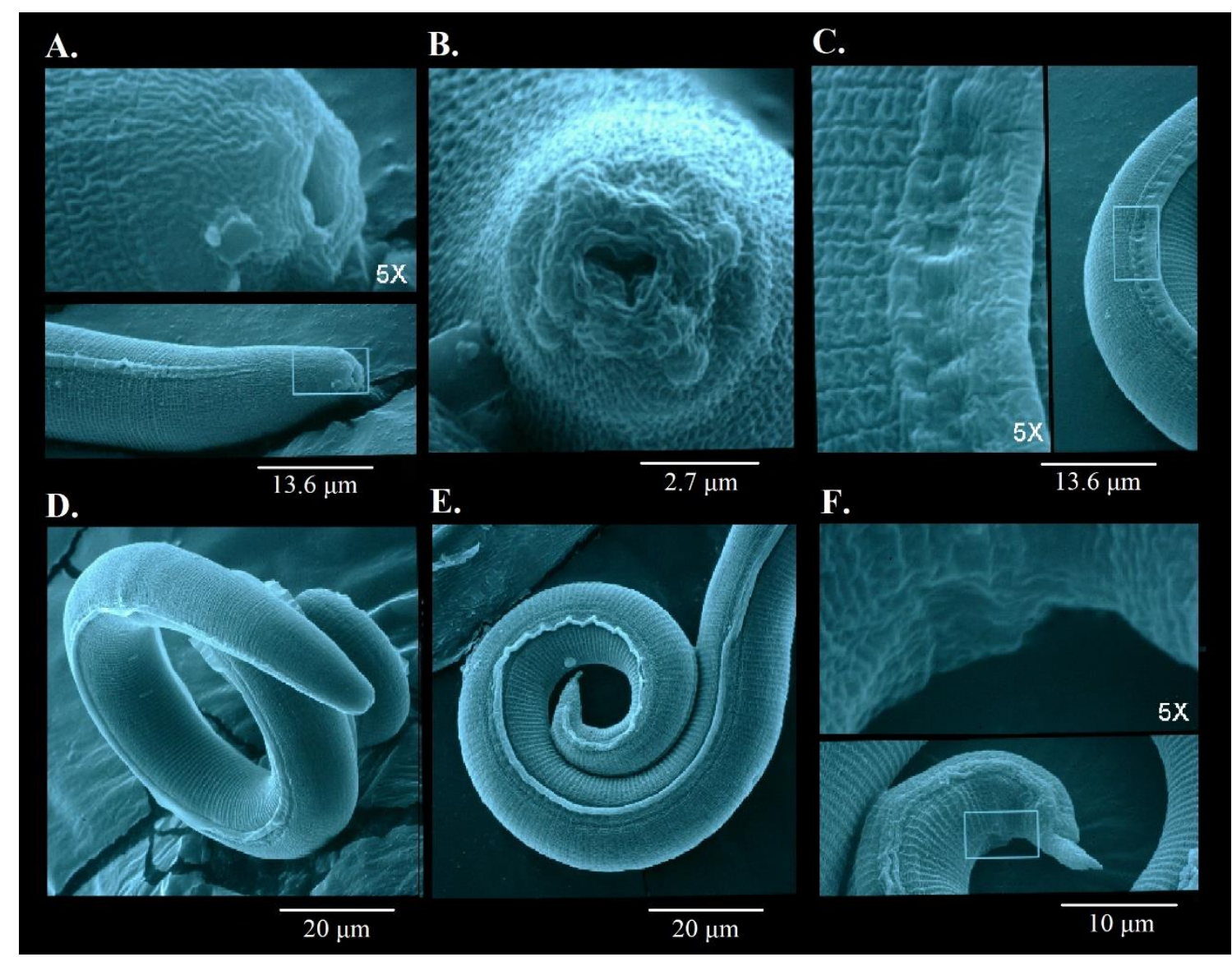

Figura 5. Larvas L3 de Angiostrongylus costaricensis A. Región anterior, se muestra una amplificación de cinco aumentos de la capsula bucal. B. Cápsula bucal C. Región media, magnificación de las expansiones longitudinales. D. Cuerpo entero larva, evidenciando expansiones longitudinales bilaterales. E. Región anterior del cuerpo, muesca menos pronunciada. F. Cauda no evidencia desarrollo de órganos sexuales.

\section{Morfología de la larva L1:}

Son larvas filiformes con una longitud de 0,26 a $0,29 \mathrm{~mm}$ y un ancho de 14 a $15 \mu \mathrm{m}$, cutícula bien desarrollada, estriaciones transversales a lo largo de todo el cuerpo y proyecciones cuticulares longitudinales, a modo de alas cefálicas extendiéndose lateralmente desde el inicio de la cauda en la muesca y a lo largo de todo el cuerpo hasta un tercio de la región anterior de la larva (Figura 6).

Hay desarrollo parcial de estructuras cefálicas, principalmente de apertura oral inmadura; pero que recuerda a la cápsula bucal del adulto. No hay clara formación de labios, papilas sensoriales y demás estructuras indicativas de desarrollo completo vistas en el adulto, aunque alrededor de la apertura oral se observan globosidades que podrían funcionar como estructuras sensoriales (Fig. 2).

En cuanto a la zona posterior, se observa la muesca en la cauda de la larva, la cual es característica de especie. No hay desarrollo de estructuras sexuales tipo, vulva o bursa copulatrix. El ano es terminal y se localiza a 26 $\mu \mathrm{m}$ del borde de la cola (Fig. 3). 


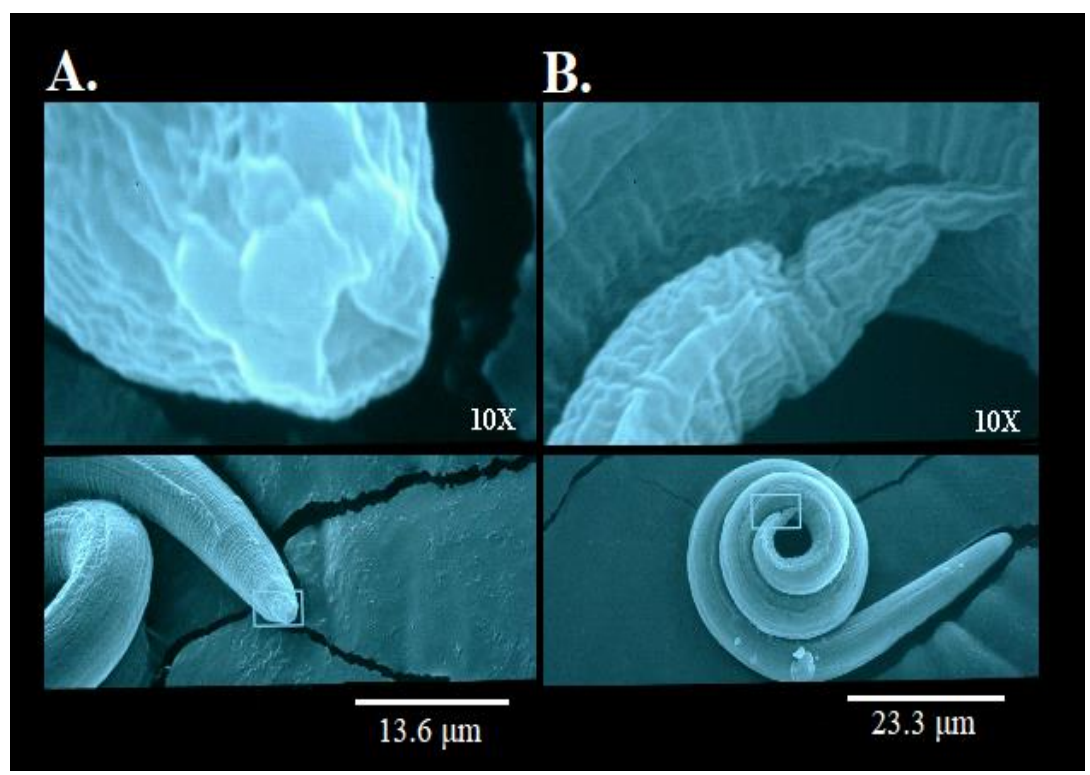

Figura 6. Larva L1 de Angiostrongylus costaricensis. A. Apertura oral mostrando un acercamiento a diez aumentos. B. Cauda con muesca característica, acercamiento a diez aumentos.

\section{Morfología de los huevecillos}

Se encontró dos tipos de morfología de huevecillos en las muestras analizadas, siendo las principales diferencias entre ellos de tipo morfológicas y morfométricas. Se visualizó que la estructura interna de la cubierta del huevecillo es distinta de la interna, siendo externamente muy texturizada e internamente prácticamente lisa, con muy leves estriaciones transversales.

Morfotipo 1: Huevecillo esférico con una cubierta muy texturizada, y de menor tamaño que el morfotipo 2. Se encontró predominio de estos en el día tres de oviposición de la hembra en cultivo de mantenimiento, con una relación aproximada de cuatro a uno.

Morfotipo 2: Huevecillo ovoidal muy aplanado en la región central y de polos redondeados. También presenta una cubierta muy texturizada. El predominio de estos se dio en el día uno de recolección, manteniendo una relación aproximada de tres a uno, con respecto al morfotipo 1. 


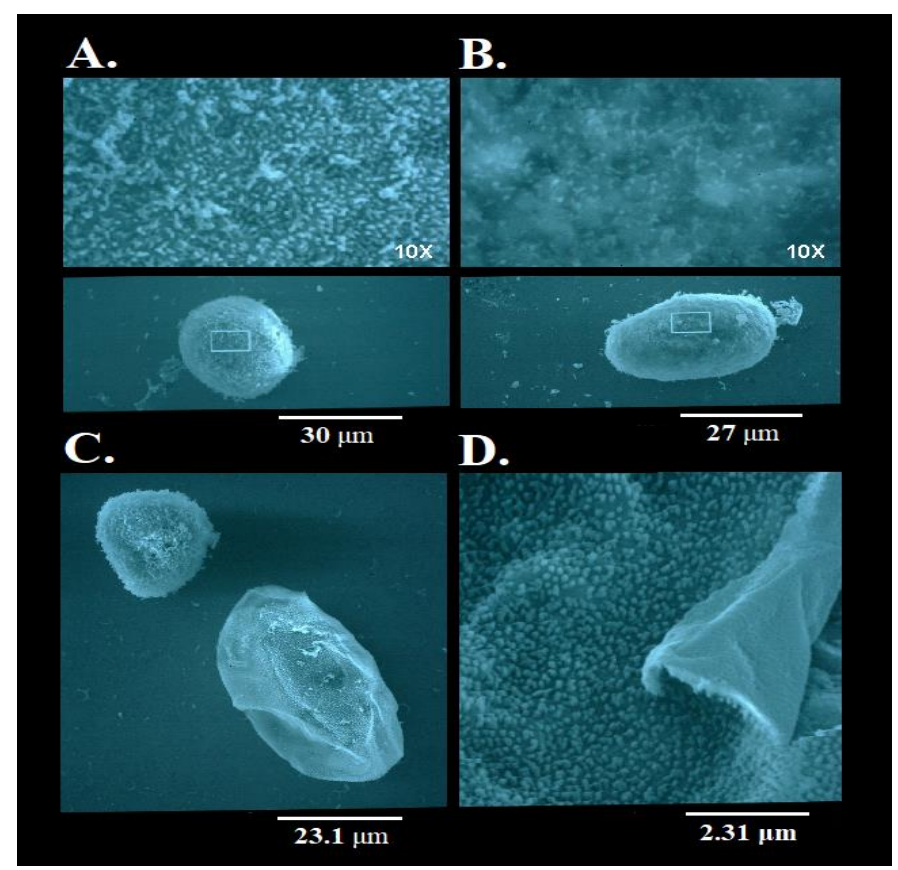

Figura 7. Huevecillos de Angiostrongylus costaricensis A. Huevecillo morfotipo uno (inmaduro). B. Huevecillo morfotipo dos (maduro). C. Morfotipos uno y dos, ver relación de tamaño. D. Corte de la cubierta mostrando su lado interno.

\section{Discusión}

El rayo externolateral de A. costaricensis, al igual que el de A. morerai, se distingue de otras especies del género, principalmente, en que los rayos mediolaterales y posterolaterales están fusionados en la mayoría de su longitud. Sin embargo, en $A$. costaricensis el rayo dorsal siempre es más corto que el rayo externodorsal y las ramas de los rayos dorsales no son tan largas como en A. morerai (Fig. 2). En todas las otras especies (excepto en $A$. morerai) los rayos posterolateral y mediolateral están únicamente fusionados en una cuarta parte o en la mitad de su longitud. En todas las especies (excepto en A. dujardini y algunas veces en A morerai) el rayo dorsal es más corto que el externodorsal. Adicionalmente, en todas las demás especies (excepto en $A$. morerai) las ramas del rayo dorsal consisten en dos o más muy cortas digitaciones.

Las espículas de A. costaricensis, por su parte, se observaron más cortas (392-420 $\mu$ m) que las de A. cantonensis $(1000-1420 \mu \mathrm{m})$, A. malaysiensis $(800-1200 \mu \mathrm{m})$ y A. chabaudi $(540 \mu \mathrm{m})$ y A. tateronae $(500-620 \mu \mathrm{m})$, pero más grandes que las de $A$. sandarsae $(330-360 \mu \mathrm{m})$, A. petrowi $(362-371 \mu \mathrm{m})$, A. ryjikovi $(320 \mu \mathrm{m})$, A. siamensis $(339 \mu \mathrm{m})$, y A. schmidti $(215-279 \mu \mathrm{m})$. Se ha descrito que otras cinco especies (A. morerai, A. dujardini, A. mackerrasae, A. sciuri, y A. vasorum) presentan longitud de las espículas en el mismo rango general que $A$. costaricensis. Morera había descrito, en especímenes obtenidos a partir de Sigmodon hispidus en Costa Rica (Morera 1973), la longitud de las espículas, las cuales reportó en un intervalo de 318-333 $\mu \mathrm{m}$. Graeff-Texeira y su grupo, infectaron ratones blancos con larvas de A. costaricensis y reportaron espículas con longitudes de 390-420 $\mu \mathrm{m}$ (Graeff Teixeira et al. 1989). Aunque hay un ligero sobrelapamiento en el rango de longitud de las espículas, entre $A$. costaricensis y A. morerai, estas especies difieren en la morfología del rayo dorsal (corto y con 2-3 pequeñas digitaciones en $A$. costaricensis) y la presencia de una pequeña espiga sobre la cola de la hembra de A. costaricensis (ausente en A. morerai).

Con respecto a los estadios larvales se ve que los cambios son básicamente en tamaño, el cual aumenta conforme la larva se acerca a su estado adulto. Llama la atención los abultamientos prominentes en la fase larval L3, visibles desde la L2, los cuales se ha explicado en publicaciones por el Dr. Morera se deben a la invasión en el hospedero intermediario (Morera 1973). Otro de los cambios importantes en el desarrollo son la aparición de expansiones a modo de alas en el estadio L3, las cuales son claramente visibles, y se piensa de pueden ser de ayuda para adhesión y movilidad en el hospedero intermediario y más tarde en el medio ambiente, ya que la L3 
es la forma infectante. Todos los huevecillos, sin excepción, mostraron en microscopía electrónica una superficie muy lisa y uniforme. Esto se debe a que la etapa de desarrollo de renacuajo es poco móvil dentro del huevecillo y no causa presión que pueda deformar la delgada pared del huevo. Y como se almacenaron en alcohol de 70 no se pudo completar su desarrollo. Si se hubiera permitido el desarrollo o se hubieran tomado huevecillos más cercanos a eclosionar se pudieron haber visto deformaciones en los huevos, producto de la movilidad de la larva embrionada en su interior.

\section{Conclusión}

En conclusión, se describió la ultraestructura de A. costaricensis en los diferentes estadios evolutivos, lo cual permite tener una idea clara de la evolución morfológica externa de este parasito a lo largo de su ciclo de vida. Se podrían hacer estudios más detallados que incluyan más muestras de cada estadio en diferentes tiempos de la evolución de cada uno de ellos, para así poder apreciar no solo un punto en la evolución del estadio sino la secuencia en el desarrollo de cada estadio.

\section{Referencias}

Abdallah, S; Elhajj, I. H; Chehab, A and Kayssi, A. (2018). A Network Management Framework for SDN. Proceedings of 9th IFIP International Conference on New Technologies, Mobility and Security (pp. 14) Paris, Francia: NTMS

Abrahams-Sandí E, Michael Geiger S, Fernández-Quesada K, S.-K. H. (2005). Specific antibody production against different life cycle stages during an experimental A. costaricensis infection in mice. Revista Biomédica, $16(4)$

239-246. https://www.researchgate.net/publication/328441421_Produccion_de_anticuerpos_especificos_contra_ diferentes_estadios_del_ciclo_de_vida_de_Angiostrongylus_costaricensis_durante_una_infeccion_exp erimental

Banevicius, N. M. S., Zanotti-Magalhães, E. M., Magalhães, L. A., \& Linhares, A. X. (2006). Behavior of Angiostrongylus costaricensis in planorbids . Brazilian Journal of Biology, Vol. 66, pp. 199-204. scielo . https://www.scielo.br/scielo.php?pid=S1519-69842006000200003\&script=sci_abstract

Geiger, S. M., Hoffmann, W. H., Soboslay, P. T., Pfaff, A. W., Graeff-Teixeira, C., \& Schulz-Key, H. (2003). Angiostrongylus costaricensis infection in C57BL/6 mice: MHC-II deficiency results in increased larval elimination but unaltered mortality. Parasitology Research, 90(5), 415-420. https://doi.org/10.1007/s00436-003-0853-2 https://pubmed.ncbi.nlm.nih.gov/12750994/

Graeff Teixeira, C., Thomé, J. W., Pinto, S. C. C., Camillo-Coura, L., Lenzi, H. L., Graeff Teixeira, C., ... Lenzi, H. L. (1989). Phillocaulis variegatus: an intermediate host of Angiostrongylus costaricensis in south Brazil. Memórias Do Instituto Oswaldo Cruz, 84(1), 65-68. https://doi.org/10.1590/S007402761989000100012 https://pubmed.ncbi.nlm.nih.gov/2319952/

Ishih, A., Rodriguez, B. O., \& Sano, M. (1990). Scanning electron microscopic observations of first- and thirdstage larvae and adults of Angiostrongylus costaricensis. The Southeast Asian Journal of Tropical Medicine and Public Health, 21(4), 568-573. https://pubmed.ncbi.nlm.nih.gov/2098918/

Morera, P. (1973). Life history and redescription of Angiostrongylus costaricensis Morera and Cespedes, 1971. The American Journal of Tropical Medicine and Hygiene, 22(5), 613-621. https://doi.org/10.4269/ajtmh.1973.22.613

http://www.ajtmh.org/content/journals/10.4269/ajtmh.1973.22.613

Morera, Pedro, \& Céspedesu, R. (2002). Angiostrongylus costaricensis n. sp. (Nematoda: Metastrongyloidea), a new lungworm occurring in man in Costa Rica . Revista de Biología Tropical , Vol. 50, pp. 783-796. scielo . https://www.scielo.sa.cr/scielo.php?script=sci_arttext\&pid=S0034-77442002000200034

Rivera, Anrabel Araya; Lobo, Lucia Quesada; Vargas, Hernando Vargas. 2015. "Angiostrongilosis Abdominal." Revista Medica de Costa Rica y Centroamerica LXXI. https://www.binasss.sa.cr/revistas/rmcc/617/art04.pdf

Sanchez, E., \& Espinoza, A. M. (2005). Ultrastructure of Oryza glumaepatula, a wild rice species endemic of tropical America. Revista de Biologia Tropical, 53(1-2), 15-22. https://www.scielo.sa.cr/scielo.php?script=sci_abstract\&pid=S0034-

$77442005000100003 \& \operatorname{lng}=$ en\&nrm=iso 
Stokes, V. L., Spratt, D. M., Banks, P. B., Pech, R. P., \& Williams, R. L. (2007). Occurrence of Angiostrongylus species (Nematoda) in populations of Rattus rattus and Rattus fuscipes in coastal forests of south-eastern Australia. Australian Journal of Zoology, 55(3), 177-184. https://doi.org/10.1071/ZO06061 https://www.researchgate.net/publication/248902275_Occurrence_of_Angiostrongylus_species_Nema toda_in_populations_of_Rattus_rattus_and_Rattus_fuscipes_in_coastal_forests_of_southeastern_Australia

Valente, R., Robles, M. del R., Navone, G. T., \& Diaz, J. I. (2018). Angiostrongylus spp. in the Americas: geographical and chronological distribution of definitive hosts versus disease reports . Memórias Do Instituto Oswaldo Cruz, Vol. 113, pp. 143-152. scielo . https://www.scielo.br/scielo.php?pid=S0074$02762018000300143 \&$ script $=$ sci_arttext\&tlng=en 